\title{
Convergence as a cue to depth'
}

WHITMAN RICHARDS ${ }^{2}$ AND JOHN F. MILLER, JR. MASSACHUSETTS INSTITUTE OF TECHNOLOGY

A test that presents conflicting monocular and vergence cues to depth shows that approximately two-thirds of the population can use convergence as a cue to depth. The remaining one-third apparently cannot use this cue. These differences in the role of convergence in depth perception reflect individual differences in the neural mechanisms underlying depth perception.

The literature is contradictory on whether the vergence position of the eyes may be used as a reliable cue to depth. Wundt (1862) performed an experiment on himself which convinced him that convergence can serve as a cue to depth in the absence of other cues. Later Es, for example Hillebrand (1893), questioned Wundt's conclusions. After reviewing the literature, Irvine and Ludvigh (1936) and Ogle (1962) concluded that depth localization from convergence alone is "impossible" or at best very unreliable. Challenging this conclusion, however, is Gogel's (1961) observation that about half his Ss were able to perceive changes in absolute distance by means of changes in convergence. Gogel's finding, taken together with the conflicting evidence in the literature, suggests that some people may be able to use convergence as a cue to distance, whereas others apparently cannot.

A second reason for the present ambiguity is that previous Es have attempted to isolate the vergence cue alone, with all other cues to depth eliminated. Such total isolation of the vergence cue is difficult to achieve. Thus, any results favoring convergence as a cue to depth were still questionable because of the possibility that extraneous cues other than vergence may still have been present.

Rather than attempt to isolate the convergence cue, the test described here specifically includes not only cues for convergence, but also other cues to depth, such as size and intensity of a "point" source in the dark. However, these "extraneous" cues all indicate that the object is at a distance different from that given by the convergence cue. The dissociation is achieved by forming a virtual image of the test light at a distance different from its true distance. For example, if the test light is located $200 \mathrm{~cm}$ from the $O$, then appropriate prisms and lenses can be placed before the $O$ 's eyes to create a virtual image of the test light at a distance of $50 \mathrm{~cm}$. In other words, to fixate properly and to see the test light in focus, the $\mathrm{O}$ must converge and accommodate as if the object were located at $50 \mathrm{~cm}$. On the other hand, the intensity, size, and any extraneous disparity cues ${ }^{3}$ would all be identical to those of an object at $200 \mathrm{~cm}$. Thus, if the $O$ indicates that the test light is at $50 \mathrm{~cm}$, he must have used the convergence information and neglected the conflicting size, intensity, and extraneous disparity cues to the object's distance.

APPARATUS
The test apparatus shown in Fig. 1 is an alley with two pin-points of light (1/16 in. in diam) seen in the dark. One light is set at a fixed distance by the $E$ and serves as the test light. The second light is a movable comparison light which the $S$ sets at the same distance as the test light. The comparison light can be moved along the same radial axis as the test light, but is approximately $2 \mathrm{~cm}$ higher.

Each of these lights is viewed separately through a prism slide, which contains two pairs of apertures $1 \mathrm{in}$. in diam. One pair of apertures contains prisms and lenses; the other pair is left open, or is used to mount lenses corresponding to the S's customary ocular correction. All lenses are approximately $3 \mathrm{~cm}$ from the S's eyes.

The $S$ can move the aperture slide back and forth, first viewing the comparison light normally and then inspecting the stationary test light through the prisms and lenses. To ensure that only one light is seen through each pair of apertures, the lights are turned on and off by microswitches placed at the ends of the slide holder.

\section{PROCEDURE}

The basic task is to match successively the two pin-points of
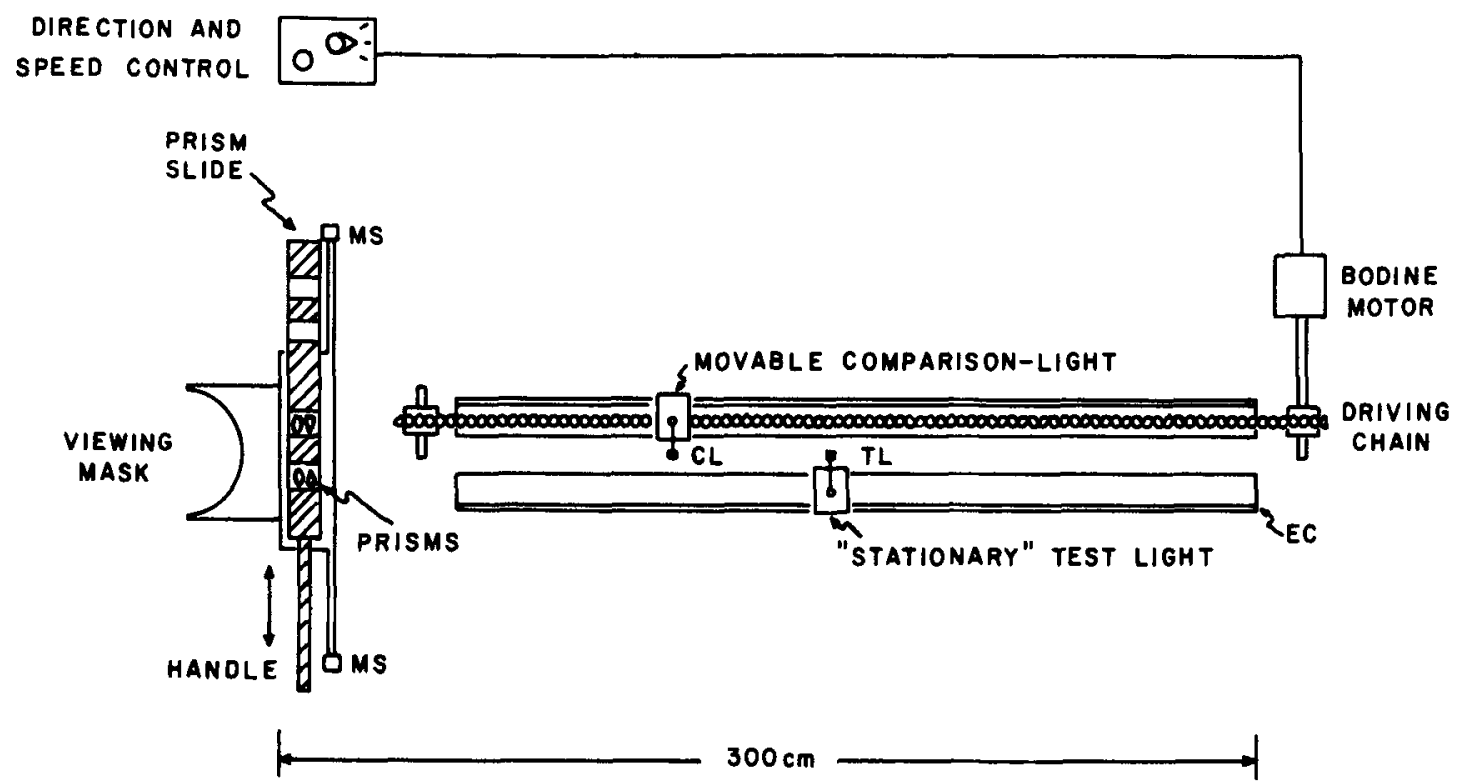

Fig. 1. Schematic diagram of the alley. TL, test light; CL, comparison light; MS, microswitch; EC, electrical contact. 
Table 1

Prism and Lens Settings for the Various Test Conditions

\begin{tabular}{cccc}
\hline Condition & $\begin{array}{c}\text { Prisms } \\
\text { (diopters) }\end{array}$ & $\begin{array}{c}\text { Lens } \\
\text { (diopters) }\end{array}$ & $\begin{array}{c}\text { Fixed Light } \\
\text { Distance (cm) }\end{array}$ \\
\hline A & +12 (B.O.) & -3 & 200 \\
B & +8 (B.O.) & -2 & 67 \\
C & +8 (B.O.) & 0 & 67 \\
D & +8 (B.O.) & -4 & 67 \\
E & -4 (B.I.) & +1 & 67 \\
\hline
\end{tabular}

light seen in the dark. For the first test, the test light is set at a fixed distance of $200 \mathrm{~cm}$ and is viewed through a pair of Risley prisms and lenses which create a virtual image at approximately $30 \mathrm{~cm}$. The comparison light, however, is observed without such prisms and with the S's normal ocular corrections. The comparison light, which is viewed naturally, can be moved by the $\mathrm{S}$ himself. His task is to move this light so that its distance away appears to him the same as the stationary test light. He sees only one light at a time in the otherwise dark room.

\section{SUBJECTS}

With four exceptions, Ss were under 35 years old, the majority being local university students whose ocular corrections did not exceed \pm 1 diopter.

\section{THE TEST}

In the distance-matching task, there are three principal cues to depth which the S may use: convergence of the eyes, and the relative size and intensity of the lights. ${ }^{3}$ The test is designed so that the depth indicated by convergence is not the same as the depth suggested by the relative sizes or intensities of the two lights. This dissociation between convergence and size or intensity is accomplished by an appropriate selection of prisms and lenses. For the first test, the prisms were set at +12 diopter (base-out) and a -3 diopter lens was added before each eye. The normal viewing condition remains unchanged throughout the tests. With the prism and lenses so set, the fixed-lamp at $200 \mathrm{~cm}$ is now optically placed at $28.5 \mathrm{~cm}$. However, the size and intensity of the light is unchanged. ${ }^{4}$ Consequently, a person using either size or intensity as cues will set the variable lamp near $200 \mathrm{~cm}$, whereas a $\mathrm{S}$ who is aware of the virtual image position will set the variable lamp to $28.5 \mathrm{~cm}$. In the case in which the variable lamp is set at $28.5 \mathrm{~cm}$, the $\mathrm{S}$ must have used convergence as a cue to depth.

As a further test for the role of convergence, several other prism and lens settings were used in addition to the one described above, as summarized in Table 1. The initial test conditions began with the movable comparison light set near $70 \mathrm{~cm}$. The starting position for each subsequent test condition was the final setting of the previous condition. The Ss were instructed to "bracket" the estimated distance before making their final setting for each test.

\section{RESULTS}

Fifteen of the 25 Ss always set the comparison light nearer to the virtual-image distance of the test light. That is to say, approximately two-thirds of the Ss appeared to use the convergence information instead of the size, intensity, and extraneous disparity cues. None of the Ss vacillated between the virtual- and real-image distance, even when they lacked confidence in their settings. There seemed to be one, and only one, correct region of appropriate distance for each person, depending upon whether or not he was able to use convergence as a cue to depth. Thus, a plot of each S's average setting shows a bimodal tendency, with each $\mathrm{O}$ consistently falling near one or the other mode. One of the modes is near the virtual-image position, whereas the second is nearer to the true position, as shown in Fig. 2 for Conditions A, B, C, and D. The distribution for Condition $\mathrm{E}$ was more ambiguous, because three of those Ss who had not previously responded to the convergence cue now shifted their distance settings to the virtual-image position. Because all the reversals for Condition $\mathrm{E}$ were accompanied by an abnormal amount of blurring of the test light, accommodative factors may have influenced the judgments. This discrepancy needs further study.

If Condition $\mathrm{E}$ is neglected for the three ambiguous Os, all the Ss can be conveniently divided into two groups: those who consistently placed the comparison light near the virtual-image position of the test light, and those who placed the comparison light at the real-image distance. For convenience, the first group is labelled Type $\mathrm{V}$, and the second group Type $R$. The mean settings for the 25 Ss, divided into their respective groups, are summarized in Table 2 . The standard deviations refer to the variance for the average settings between Os. The standard deviation for any one match made by a $S$ ranged from 0.25 to 0.5 diopter.

A closer look at Table 2 shows that Conditions C and D used lenses that were not normally appropriate for the prismatic deviation For example, in Condition C, a S whose eyes were fixating the test light would be converging toward a point at $28.5 \mathrm{~cm}$, but would need to accommodate to $67 \mathrm{~cm}$. Similarly, in Condition D, the convergence position was again at $28.5 \mathrm{~cm}$, but accommodation is as if the test light were placed at $18 \mathrm{~cm}$. These conditions show whether convergence or the stimulus to accommodation was the relevant cue to
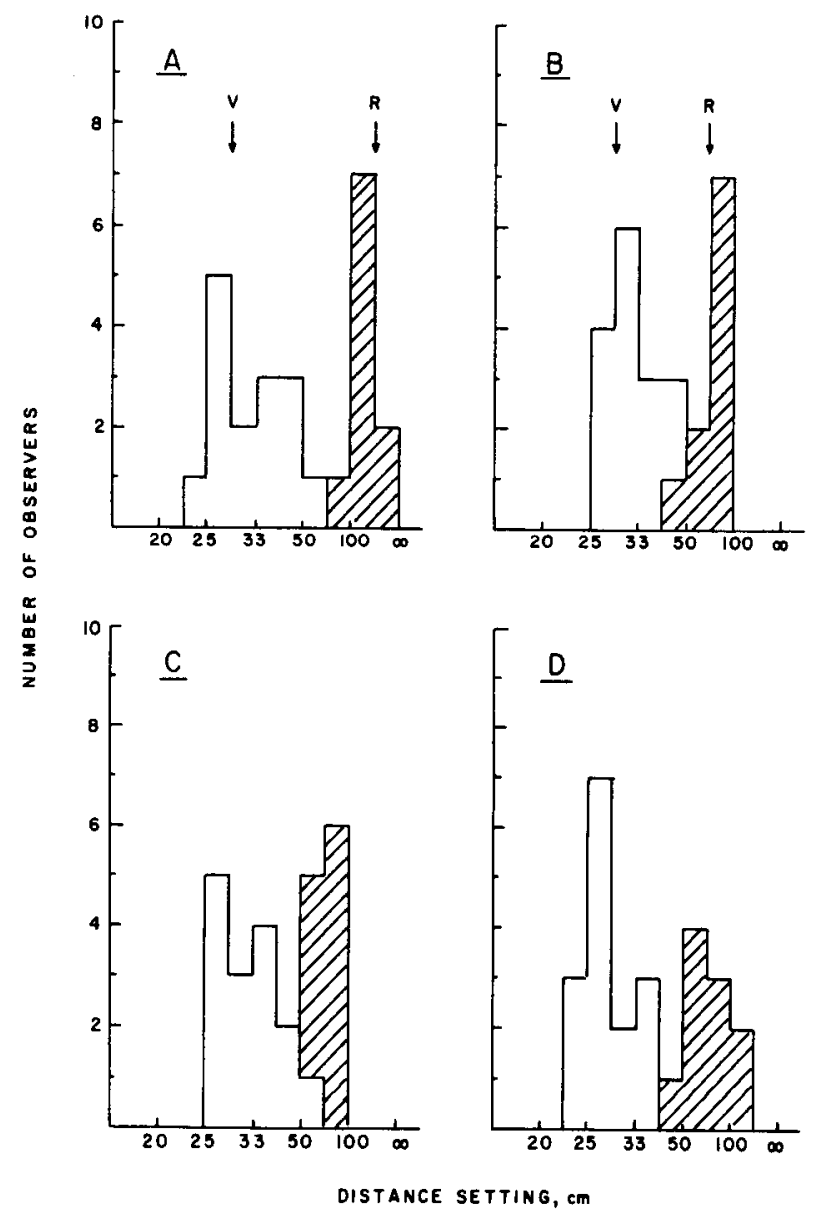

Fig. 2. Distributions of the Os' distance settings for the first four test conditions summarized in Tables 1 and 2. The virtual- and real-image distances are indicated by the arrows. The cross-hatching indicates those Os whose average distance setting for all conditions is nearest to the real-image distance. 
Table 2

Distance Settings in Centimeters for the Five Test Conditions

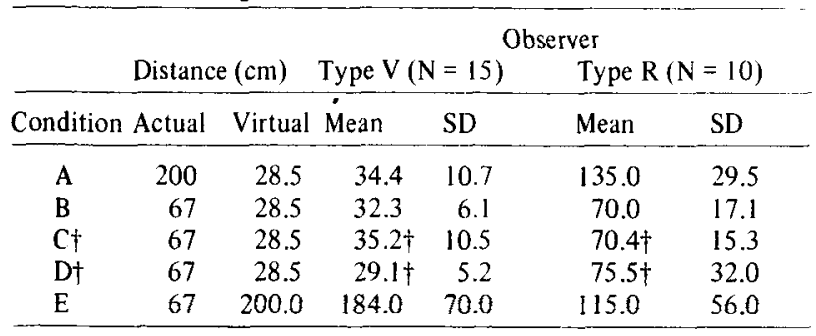

t Accommodation and convergence are mismatched for these conditions. See Table 1 .

depth when the virtual image was "sensed." Because the mean settings remained near $30 \mathrm{~cm}$, corresponding to the approximate convergence position of the test light, convergence and not the accommodative stimulus must have been the more important cue. This result confirms a similar conclusion reached by Swenson (1932), using a different method.

\section{DISCUSSION}

When a $\mathbf{S}$ uses convergence as a cue to depth, he still has available the cues of size and intensity. Apparently these latter cues were ignored by the Type V S. By the same reasoning, the Type R Ss who did not use the convergence cue may still have had the convergence information available, but may have chosen to ignore it. Consequently, the proportion of Ss who are able to use vergence as a cue to depth may be underestimated. The error of underestimation is undoubtedly low for two reasons.

First, no $\mathrm{S}$ reported ambiguity concerning where the variable light should be set. For each $S$ there was one and never two possible regions where the variable light should be set for a match. The judgment became ambiguous for the Type V Os only when additional disparity information was provided by introducing a third light seen at a fixed distance under both the comparison and test conditions. This third light provided a strong cue to the relative distances of the test and comparison lights, thereby eliminating the need for abbolute judgments of depth. With the third light on, the additional disparity information conflicted with the convergence information, and the final setting depended on whether the $\mathrm{S}$ judged absolute or relative distance.
The second reason why minimal errors have probably been made in identifying those Ss who could use convergence as a cue to deptl is based upon correlations with fixation-disparity curves. Fixation disparity refers to the angular displacement of two fused identical images which are being fixated. This displacement occurs particularly when convergence and its normal corresponding stimulus for accommodation are altered. Fig. 3 shows such curves for two Ss, derived according to Ogle's method (1964). ${ }^{5}$ The observation distance was always $200 \mathrm{~cm}$. Along the abscissa are various settings of the prisms through which the $S$ viewed the pattern. The ordinate shows the actual discrepancy between the fixation positions of his two eyes for any given prismatic condition. The addition of a -3 diopter lens displaces these fixation-disparity curves to the right by about 6 to 8 prism diopters. This displacement is not unexpected, because additional prism power (ideally +12 diopters) would be necessary to produce the amount of convergence that is normally appropriate for +3 diopters of accommodation.

Nevertheless, introducing an accommodative bias of +3 diopters does not always bring about a corresponding lateral shift in the fixation disparity curve, as shown in Fig. 4. The results obtained from these Os are typical of a second $k$ ind of fixation-disparity data, which show that the addition of a lens may have very little effect.

The two different kinds of behavior revealed by the fixation-disparity curves in Figs. 3 and 4 are to a large extent related to the S's behavior on the depth-matching task. Those Ss who did not use the convergence cue (Type R) generally had fixation-disparity curves that were laterally shifted to the right after introducing a -3 diopter lens (similar to Fig. 3). On the other hand, those Os who used the convergence cue (Type V) generally had fixation-disparity curves that were almost unchanged after addition of the -3 diopter lens. (Part of the Type V group also showed vertical shifts.) These correlations are summarized in Table 3.

The correlations between the behavior of the fixationdisparity curves and the S's ability to use convergence as a cue to depth suggest that the basic difference between the Type $R$ and Type V Os may be neurogenic. Fixation-disparity curves are the "finger prints" of the oculomotor system; they reflect an interaction between oculomotor imbalance and sensory fusion. Curves of fixation disparity remain relatively stable over time and do not appear modifiable by "learning" (Ogle, Martens, \& Dyer, 1967). Thus the correlations shown in


Fig. 3. Fixation-disparity curves for two Type $R$ Os under two accommodative conditions, showing the lateral shift of the curve after adding $\mathbf{- 3}$ diopter lenses. 



Fig. 4. Same as Fig. 3, except for two Type V Os showing that the addition of -3 diopter lenses has hardly altered the fixation curve.

Table 3 suggest that the ability to use convergence as a cue to depth may be an inborn aptitude, reflecting a property of the individual's mechanism for depth perception.

Table 3

Observers Grouped According to Their Ability to Use Convergence as a Cue to Depth and the Behavior of Their Fixation-Disparity Curves.

Effect on the fixation-disparity curve of introducing a -3 dipter lens

\begin{tabular}{lcc}
\multicolumn{1}{c}{ introducing a -3 dipter lens } & Observer & Type \\
\hline & V & R \\
Shifted horizontally & 3 & 9 \\
Stable or shifted vertically & 9 & 1 \\
\hline
\end{tabular}

$\chi^{2}=8.2 ; p<.01$

\section{REFERENCES}

GOGEL, W. C. Convergence as a cue to absolute distance. Report No. 467, U.S. Army Medical Research Laboratory, Fort Knox, Kentucky, 1961.

HILLEBRAND, F. Die Stabilität der Raumwerte auf der Netzhant. Zeitschrift für Psychologie und Physiologie Sinnesorg., 1893, 5, 1-60.

IRVINE, S. R., \& LUDVIGH, E. J. Is ocular proprioceptive sense concerned in vision? Archives of Ophthalmology, 1936, 15, $1037-1049$.

OGLE, K. N. Perception of distance and size. In H. Davson (Ed.), The eye. Vol, 4: Visual optics and the optical space sense. New York: Academic Press, 1962. Chap. 14.

OGLE, K. N. Researchès in binocular vision. New York: Hafner, 1964.

OGLE, K. N. Visual space perception. In C. H. Graham (Ed.), Vision and visual perception. New York: John Wiley, 1965. Pp. 504-547.
OGLE, K. N., MARTENS, T. G., \& DYER, J. A. Oculomotor imbalance in binocular vision. Philadelphia: Lea and Febiger, 1967.

SWENSON, H. A. The relative influence of accommodation and convergence in the judgment of distance. Journal of General Psychology, 1932, 7, 360-380.

WUNDT, W. Beitrage zur Theorie der Sinneswahrnehmungen. Leipzig and Heidelberg, 1862. Pp. 105-134, 12-199.

\section{NOTES}

1. Supported by the U.S. Air Force under Contract No. AFOSR-F44620-67-C0085.

2. Address: Department of Psychology, Massachusetts Institute of Technology, Cambridge, Mass. 02139.

3. If dark adaptation proceeded for about $5 \mathrm{~min}$, the light from the test lamps that was reflected off the base of the prisms became slightly visible in the periphery of the eye. This scattered light provided a disparity cue to the real distance of the test light, thereby reinforcing the size and intensity cues. To minimize this disparity cue, field stops of different sizes were placed in front of the left and right apertures.

4. Because the prisms and lenses absorb some light, the intensity is reduced very slightly. In addition, the lenses alter the image of the test lamp, depending upon their power and their distance from the eye. For a -3 diopter lens, the image size is reduced by about $10 \%$ for our test conditions.

5. This method is described completely on p. 72 of Ogle's book, Researches in binocular vision (1964). The only alteration was that we used an opaque, central square $2 \mathrm{deg}$ on a side which was located in the middle of a 10-deg wide acuity chart. The luminance of the projected chart was $6.5 \mathrm{ft}-\mathrm{L}$.

(Accepted for publication November 18, 1968.) 